\title{
BMJ Open Diagnostic yield of chest and thumb ECG after cryptogenic stroke, Transient ECG Assessment in Stroke Evaluation (TEASE): an observational trial
}

\author{
Peter Magnusson (D) , ${ }^{1,2}$ Adam Lyren, ${ }^{1}$ Gustav Mattsson ${ }^{1}$
}

To cite: Magnusson $P$, Lyren A, Mattsson G. Diagnostic yield of chest and thumb ECG after cryptogenic stroke, Transient ECG Assessment in Stroke Evaluation (TEASE): an observational trial. BMJ Open 2020;10:e037573. doi:10.1136/ bmjopen-2020-037573

- Prepublication history for this paper is available online. To view these files, please visit the journal online (http://dx.doi. org/10.1136/bmjopen-2020037573).

Received 21 February 2020 Revised 05 August 2020 Accepted 12 August 2020
Check for updates

(C) Author(s) (or their employer(s)) 2020. Re-use permitted under CC BY-NC. No commercial re-use. See rights and permissions. Published by BMJ.

${ }^{1}$ Centre for Research and Development, Uppsala University/Region Gävleborg, Uppsala Universitet, Uppsala, Sweden

${ }^{2}$ Institution of Medicine, Karolinska Institute, Stockholm, Sweden

Correspondence to Dr Peter Magnusson; peter.magnusson@ regiongavleborg.se

\section{ABSTRACT}

Objective In stroke survivors, atrial fibrillation (AF) is typically evaluated solely by short-term ECG monitoring in the stroke unit. Prolonged continuous ECG monitoring or insertable cardiac monitors require substantial resources. Chest and thumb ECG could provide an alternative means of AF detection, which in turn could allow prompt anticoagulation to prevent recurrent stroke. The objective of this study was to assess the yield of newly diagnosed AF during 28 days of chest and thumb ECG monitoring two times per day in cryptogenic stroke.

Methods This study, Transient ECG Assessment in Stroke Evaluation, included patients who had a stroke from Region Gävleborg, Sweden, between 2017 and 2019. Patients with a recent ischaemic stroke without documented AF (or other reasons for anticoagulation) before or during ECG evaluation in the stroke unit were evaluated using the Coala Heart Monitor connected to a smartphone application for remote monitoring.

Results The prespecified number of 100 patients (mean age $67.6 \pm 10.8$ years; $60 \%$ men) was analysed. In nine patients $(9 \%$, number needed to screen 11$) \mathrm{AF}$ but no other significant atrial arrhythmias (>30 s) was diagnosed. The mean $\mathrm{CHA}_{2} \mathrm{DS}_{2}$-VASc score was similar among patients with $A F$ and no AF ( $4.9 \pm 1.1$ vs $4.3 \pm 1.3 ; p=0.224)$ and patients with AF were older (74.3 \pm 9.0 vs $66.9 \pm 10.8$; $p=0.049$ ). Patients performed on average $90.1 \% \pm 15.0 \%$ of scheduled transmissions.

Conclusion In evaluation of cryptogenic stroke, $9 \%$ of patients had AF detected using chest and thumb ECG two times per day during 1 month. In many stroke survivors, this is a feasible approach and they will be potentially protected from recurrent stroke by anticoagulation treatment.

Trial registration number NCT03301662.

\section{INTRODUCTION}

Stroke is a leading cause of death, disability and morbidity including dementia. ${ }^{1}$ The global burden of stroke remains a challenge due to ageing populations and the growing prevalence of risk factors, such as congestive heart failure, hypertension, diabetes, vascular disease and the interplay with atrial fibrillation $(\mathrm{AF}) .^{23} \mathrm{AF}$ is a complex condition known to cause stroke and systemic
Strengths and limitations of this study

- Prospective study design.

- Evaluation of atrial fibrillation using a combined chest and thumb ECG after stroke.

- Pragmatic approach to evaluation of cryptogenic stroke that increases generalisability.

- Use of the Coala Life Monitor which is connected to a web-based portal.

- Limited sample size based on stroke units at two centres.

embolisation, but these devastating events can be prevented by anticoagulant therapy. ${ }^{4}$ A non-vitamin $\mathrm{K}$ antagonist oral anticoagulant (NOAC) is the preferred choice because of superior efficacy, lower risk of bleeding and fewer interactions. ${ }^{5}$ A meta-analysis of the pivotal NOAC trials showed a $19 \%$ reduction of stroke/systemic embolism and a $10 \%$ lower mortality compared with warfarin. ${ }^{5}$ If AF is not diagnosed, then antiplatelet medication is the current practice following a stroke. ${ }^{67}$ According to the European Society of Cardiology (ESC), antiplatelet monotherapy should not be considered in the presence of AF, regardless of the stroke risk. ${ }^{67}$ At least $20 \%-30 \%$ of patients with ischaemic stroke have a documented episode of $\mathrm{AF}$ before, during or after the stroke, but in a quarter of patients, the stroke is cryptogenic, meaning that no attributable cause can be discerned. ${ }^{8-10}$

The strategies for AF detection after stroke include monitoring using continuous ECG in the hospital ward, repeated ECGs, Holter monitoring, external event or loop recorders, long-term monitoring using patches and handheld devices. Insertable cardiac monitors in cryptogenic stroke yield an AF diagnosis in $8.9 \%$ at 6 months and $12.4 \%$ at 12 months, but this invasive strategy has not been endorsed in routine care; it requires 
considerable resources and implies high upfront costs, even though it seems to be cost-effective. ${ }^{11}{ }^{12}$ Episodes of AF may be silent, thus not recognised or reported by the patient, but are nevertheless associated with the same risk of embolisation. ${ }^{13-15}$ In patients with either dual-chamber pacemakers or implantable defibrillators and with no documented history of AF, atrial high rate episodes are associated with an increased risk of stroke, although the temporal aspects suggest complex causative pathways. ${ }^{1617}$ The cut-off duration for increased risk among patients with implantable devices is controversial, but episode duration of more than 1 hour doubles the risk for ischaemic stroke. ${ }^{18-20}$ The current position of the ESC clearly advocates a low threshold for NOAC in patients with multiple risk factors and especially in secondary prevention of stroke. ${ }^{21}$ A handheld ECG device typically monitors for $30 \mathrm{~s}$ to meet the definition of AF, but captured episodes often reflect longer and repeated episodes and have become a widely accepted indication to initiate NOAC in newly detected $\mathrm{AF}^{6}{ }^{6} 7$ Given the fact that prior stroke or transient ischaemic attack (TIA) is the most powerful risk factor for recurrent stroke because it confers a $10 \%$ per year risk, there is clearly an urgent need for accurate detection of $\mathrm{AF}$ and prompt therapeutic intervention to prevent recurrent stroke. ${ }^{22}$

Sequentially stratified ECG monitoring detected AF in $24 \%$ of patients who had a stroke. ${ }^{8}$ The diagnostic yield was $11.5 \%$ in a pooled analysis, but this yield varies with such factors as timing, length of registration and the monitoring tool. ${ }^{23}$ Thus, while ECG monitoring over an extended period of time is crucial for stroke survivors, post-discharge Holter monitoring or patches are impractical, ECG data storage is limited, and data interpretation requires considerable resources.

A large multicentre study of patients who had a stroke on Holter monitoring reported new diagnoses of AF in $2.6 \%$ of patients at 24 hours and $4.3 \%$ at 72 hours. ${ }^{10}$ In another study, AF was detected in $8.3 \%$ of patients who had a stroke monitored by continuous ECG for a median of 89 hours in the stroke unit; prolonged monitoring was superior to 24 hours monitoring in detecting $\mathrm{AF}^{24}$ Since ECG monitoring over an extended period of time is important for stroke survivors, the thumb ECG offers advantages: a two times per day monitoring schedule is convenient for patients, who can also use the device to capture symptomatic episodes.

The chest and thumb ECG Coala Heart Monitor (Coala Life AB, Stockholm, Sweden) uses a smartphone application for remote monitoring through a web-based platform. The system offers validated algorithms and the additional chest ECG, which allows for differentiation between arrhythmias. ${ }^{25}$ However, diagnostic yield and feasibility of this system in patients who recently suffered a stroke need to be evaluated.

The objective of this prospective study was to assess the yield of newly diagnosed AF during 28 days of chest and thumb ECG for $30 \mathrm{~s}$ in cryptogenic stroke.

\section{METHODS}

\section{Setting and selection}

Patients with a clinically confirmed diagnosis of ischaemic stroke were recruited from the catchment area of Region Gävleborg, Sweden and its two stroke units in Gävle and Hudiksvall, respectively. Eligible patients were identified from weekly checks of the medical records.

\section{Inclusion and exclusion}

Patients, aged $\geq 18$ years, with a diagnosis of ischaemic cryptogenic stroke were eligible for the study. Cryptogenic stroke is defined as cerebral ischaemia of unknown aetiology, that is, not attributable to a source of cardiac embolism, large artery atherosclerosis or small artery disease despite a standard vascular, cardiac and serologic evaluation. ${ }^{24}$ The exclusion criteria were as follows: previously known atrial tachycardia with an indication for anticoagulation; an implantable cardioverter defibrillator, pacemaker or insertable cardiac monitor; pregnancy; indication for anticoagulation (including low-molecular weight heparin) due to atrial tachycardia, mechanical heart valve, deep vein thrombosis or pulmonary embolism. Thus, if outcome is reached the patient should be a candidate for anticoagulation therapy. Patients with a life expectancy $\leq 6$ months or severe cognitive impairment were excluded. Patients with a TIA were not included, because this diagnosis is often uncertain when being based solely on patients' history. ${ }^{26}$ While the more recent tissue-based definition of TIA using magnetic resonance diffusion weighted imaging improves the diagnostic accuracy, this is not always done in routine clinical practice. ${ }^{27}$

\section{Stroke evaluation}

Patients were evaluated with a carefully taken patients' history, physical examination, routine laboratory tests and standard evaluation using 12-lead ECG, 24-hour Holter, carotid Doppler ultrasonography and/or CT angiography, CT, and, when applicable, MRI, echocardiography or transesophageal echocardiography, as well as extended coagulation tests.

\section{Outcome measurements}

$\mathrm{AF}$ or atrial flutter with a duration of at least $30 \mathrm{~s}$. The date and time of each episode were recorded.

\section{Study endpoint}

The endpoint was 28-day cumulative incidence of AF or atrial flutter.

\section{Chest and thumb ECG}

Patients were asked to use the chest and thumb ECG monitor device two times per day, once between the hours of 06:00 and 10:00 and again between 18:00 and 22:00. The monitoring was planned to start within a few days after the diagnosis of stroke had been confirmed and standard evaluation was complete.

If the patients felt palpitations or other symptoms suggestive of arrhythmia, for example, sudden onset of tiredness, dyspnoea, syncope, they were asked to record 
the episode with the smartphone application. Each patient was monitored for 4 consecutive weeks (28 days).

Each recording was stored in a web-based application accessible to the investigators. The investigators checked all recordings daily. In the case of an arrhythmia that would imply outcome, a second investigator, an experienced cardiologist within the field of arrhythmia, interpreted the recording. If the outcome was reached, anticoagulation was promptly started.

\section{Statistics}

Descriptive data are reported as frequencies, percentages, means, IQRs and percentiles. Continuous variables are summarised as means, $\mathrm{SD}$ and percentiles, and t-tests were used for group comparisons, while $\mathrm{X}^{2}$ test was used for categorical variables. Statistical significance was defined as a two-sided $\mathrm{p}$ value of $<0.05$. The data were stored in Excel V.2010 (Microsoft Corporation, Redmond, Washington, USA) and imported into SPSS V.25 for analyses.

\section{Ethics and dissemination}

The study protocol was registered at clinical trial registration and published.$^{28}$ Each patient was informed by a study physician and included after written consent.

\section{Patient and public involvement}

Patients were not involved in the design, or conduct, or reporting, or dissemination plan of our research.

\section{RESULTS}

As per protocol, 100 patients with a history of ischaemic stroke were evaluated (out of 111 who consented to participate) between October 2017 and October 2019. The median time from index stroke to inclusion was 7 days (IQR 4-13 days). The mean age at inclusion was $67.6 \pm 10.8$ years. The ages ranged from 27 to 86 years and 25th, 50th and 75th percentiles were 61.8, 69.0, 74.9 years, respectively. The majority were men $(60.0 \%)$ and the mean age was similar between men and women (66.7 \pm 9.7 vs $68.9 \pm 12.3$ years; $\mathrm{p}=0.323)$.

The mean total $\mathrm{CHA}_{2} \mathrm{DS}_{2}$-VASc score was $4.4 \pm 1.9$ points. Because stroke implies 2 points per se, all patients had a score of at least 2. No patients had 8 or 9 points and the scores were normally distributed within the cohort.

\section{Outcome}

In total, the 28 days of scheduled chest and thumb ECG yielded $9 \%(\mathrm{n}=9) \mathrm{AF}$ among the participants. An example of $\mathrm{AF}$ is shown in figure 1 (as a comparison sinus rhythm is shown in figure 2). No atrial flutter or ectopic atrial

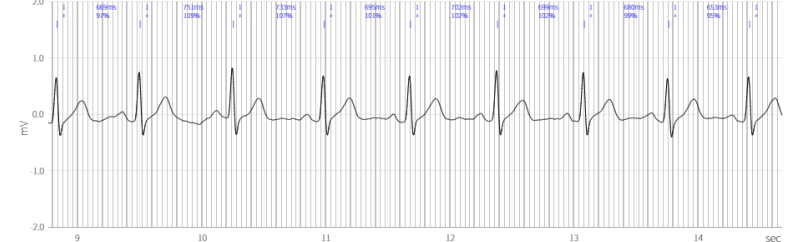

Figure 2 Example of an ECG transmission from a Coala Heart Monitor showing normal sinus rhythm.

tachycardia was diagnosed. The first episode of $\mathrm{AF}$ was diagnosed at a mean of $12.7 \pm 7.8$ days (range day $1-23$ ). The characteristics of the cohort at baseline and with regard to outcome is summarised in table 1 . The nine AF episodes were characterised by a mean frequency of $115 \pm 31$ (range 72-166) beats per min. The mean $\mathrm{CHA}_{2} \mathrm{DS}_{2}$-VASc score was similar among patients with AF and no AF (4.9 \pm 1.1 vs $4.3 \pm 1.3 ; \mathrm{p}=0.224)$ and patients with AF were older $(74.3 \pm 9.0$ vs $66.9 \pm 10.8$; $\mathrm{p}=0.049)$.

Out of nine patients with detected atrial fibrillation, one patient had come into contact with healthcare due to the episode, he reported that he experienced symptoms in the form of palpitations and dizziness the night before AF was detected. He had experienced similar symptoms previously but this time after seeing that the automated report of the scheduled ECG in the morning was abnormal, he went to the emergency department and was admitted due to rapid AF. Out of the other eight patients with detected $\mathrm{AF}$, one reported feeling palpitations, one reported feeling stressed as well as dizzy and six reported feeling well with no symptoms in conjunction with the episode.

There was no adverse event related to the use of the ECG device. No patient died during the monitoring period.

\section{Adherence to protocol}

Among the 111 participants who consented to participate, 11 dropped out because of cognitive or physical impairment that made them unable to handle the technology or they did not wish to participate. Among the 100 participants who completed the study, a total of 5249 ECG transmissions were collected during the 28-day period. Patients performed on average $90.1 \% \pm 15.0 \%$ (25th, 50th and 75 th percentiles were $87.5 \%, 96.4 \%$ and $98.2 \%$, respectively) of scheduled transmissions. The vast majority of patients $(n=86)$ performed extra transmissions (mean $0.21 \pm 0.29$ /day). The total number of extra transmission per patient was $4.8 \pm 5.2$ (range 0-24). If an episode of

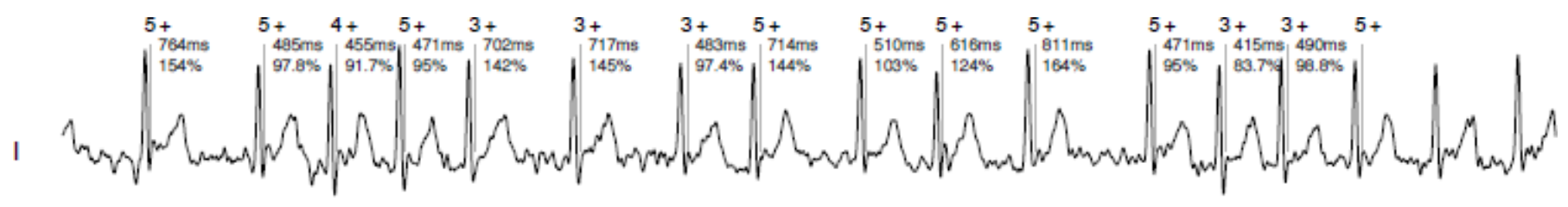

Figure 1 Example of an ECG transmission from a Coala Heart Monitor for an episode of atrial fibrillation. 
Table 1 Characteristics of 100 patients at baseline

\begin{tabular}{|c|c|c|c|}
\hline & All (\%) & No atrial fibrillation (\%) & Atrial fibrillation (\%) \\
\hline Patients & $100(100)$ & $91(91)$ & $9(9)$ \\
\hline Mean age (years) & $67.6 \pm 10.8$ & $66.9 \pm 10.8$ & $74.3 \pm 9.0$ \\
\hline Congestive heart failure & $1(1)$ & $1(1.1)$ & $0(0)$ \\
\hline Hypertension & $78(78.0)$ & $71(78.0)$ & $7(77.8)$ \\
\hline Diabetes mellitus & $19(19.0)$ & $18(19.8)$ & $1(11.1)$ \\
\hline Stroke & $100(100.0)$ & $91(100.0)$ & $9(100.0)$ \\
\hline Vascular disease & $17(17.0)$ & $16(17.6)$ & $1(11.1)$ \\
\hline Age $65-74$ years & $37(37.0)$ & $34(37.4)$ & $3(33.3)$ \\
\hline Sex category (female) & $40(40.0)$ & $34(37.4)$ & $6(66.7)$ \\
\hline 0 point & $0(0.0)$ & $0(0.0)$ & $0(0.0)$ \\
\hline 1 point & $0(0.0)$ & $0(0.0)$ & $0(0.0)$ \\
\hline 2 points & $6(6.0)$ & $6(6.6)$ & $0(0.0)$ \\
\hline 3 points & $22(22.0)$ & $22(24.2)$ & $0(0.0)$ \\
\hline 4 points & $24(24.0)$ & $20(22.0)$ & $4(44.4)$ \\
\hline 5 points & $28(28.0$ & $25(27.5)$ & 3 (33.3) \\
\hline 6 points & 15 (15.0) & $14(15.4)$ & $1(11.1)$ \\
\hline 7 points & $5(5.0)$ & $4(4.4)$ & 1 (11.1) \\
\hline 8 points & $0(0.0)$ & $0(0.0)$ & $0(0.0)$ \\
\hline
\end{tabular}

Data presented as frequencies (percentage in parenthesis).

AF was detected, the study outcome was reached and the patient's participation in the study was completed.

\section{DISCUSSION}

Prolonged monitoring using the Coala Heart Monitor detected $\mathrm{AF}$ in $9 \%$ of patients with cryptogenic stroke, all of whom received NOAC to prevent recurrent stroke. Because we targeted solely patients who were candidates for a change in medication regimen in the presence of $\mathrm{AF}$, this led to a potential benefit in the clinical management of these individual patients. The number needed to screen based on the yield in our study is 11 . The estimated risk of ischaemic stroke in patients with $\mathrm{AF}$ without anticoagulation with $\mathrm{CHA}_{2} \mathrm{DS}_{2}$-VASc score 4 is 4.8 per 100 years (6.7 if the composite stroke/TIA/systemic embolism is applied). ${ }^{29}$ Given the fact that prior stroke/TIA is the most powerful risk factor and confers a $10 \%$ per year stroke risk, the urgent need for rapid identification of $\mathrm{AF}$ and subsequent treatment after the first stroke is underscored. ${ }^{22}$

Our findings have implications for clinical practice and widespread applicability for secondary prevention of stroke. The common practice to rely on 24-48hours of monitoring as part of stroke evaluation is insufficient and should be regarded as an initial screen. Improving the detection of AF will be increasingly important in an ageing population with a considerable burden of other cardiovascular risk factors. Indeed, the worldwide cardiovascular burden demands a targeted approach to AF and its consequences. ${ }^{30}$

\section{Compared to a study using ECG chest belt}

The largest prospective study of event-triggered recordings of patients (mean age 72.5 years) with a history of cryptogenic stroke used a dry-electrode, non-adhesive, belt around the chest for 30 days to enable better compliance than skin-contact electrodes; $82 \%$ wore it at least 21 days. ${ }^{31}$ The AF yield was 12.9 percentage points absolute difference compared with 1 day of Holter ECG monitoring $(16.1 \%$ vs $3.2 \%)$.

Compared with our study, these patients were slightly older, such a belt might be better suited to patients who are unable to handle a smartphone-based system. Nevertheless, compliance among our patients was better; those who mastered the smartphone-based device are more likely to comply with the protocol because it is more convenient than wearing a belt. This effect is likely to become 
more pronounced over time. Our study has overcome two of the major limitations of this earlier study. First, the mean time from the index event of stroke to participation in our study was $11.6 \pm 13.7$ days, compared with $75.1 \pm 38.6$ days in the belt study. This late start of monitoring implies that patients were not monitored during the vulnerable initial period after stroke, which may delay the initiation of protective treatment. Indeed, most AF episodes occur around the time of the index event. However, studies using implantable devices show that the cumulative incidence of $\mathrm{AF}$ continues to increase after the first month. ${ }^{11}{ }^{19}$ Second, as the authors suggested, including patients with TIA might dilute the cohort with low-risk patients if TIA was not the correct diagnosis. This, in turn, could affect outcome. ${ }^{25}$

\section{Comparison of chest ECG}

In a Danish cohort of 95 patients with stroke/TIA, using the Zenicor handheld device two times per day for 30 days yielded 20 patients with $\mathrm{AF}$ but concurrent Holter monitoring detected 11 of these episodes during the first day. ${ }^{32}$ Thus, in 10 out of 95 patients, the thumb ECG detected AF that was not diagnosed by Holter. The median time from index event to AF diagnosis was 4 days. The mean age was 79 years, considerably higher than in our study, which may have compensated for the inclusion of TIA to result in similar yield as in our study. The finding that $13 \%$ of tracings were not usable because poor quality may reflect the older age of the study population, but also stresses the importance of thorough patient education at the time of inclusion and support during follow-up, if needed. Of all eligible patients, $22 \%$ could not be included because of cognitive and physical impairment. We share the experience that not all patients are suitable for using a thumb-ECG in this type of evaluation.

In another post stroke/TIA study $(\mathrm{n}=249)$ using thumb ECG two times per day for 30 days, the yield was $6.8 \%$ and $11.8 \%$ in patients aged 75 years and older. ${ }^{33}$ The age distribution of $\mathrm{AF}$ is in line with epidemiological data and previous findings. It should be noted that recording capabilities of the devices at that time caused the definition of $\mathrm{AF}$ to be limited to episodes of $10 \mathrm{~s}$, which is shorter than the generally accepted definition of AF episodes of $30 \mathrm{~s}$, endorsed by current guidelines. ${ }^{7}$ Moreover, patients with a suspected TIA were included, in contrast to our study. Interestingly, $94 \%$ of all detected $\mathrm{AF}$ episodes occurred during the first 20 days.

In a single-centre retrospective analysis, 13 out of 114 patients (11.4\%) with stroke/TIA had AF detected by a Zenicor ECG during a 21-day follow-up with recordings scheduled two times per day. ${ }^{34}$ Episodes of less than $30 \mathrm{~s}$ were not considered as AF. Notably, the mean age of the patients was 70.3 years and $27.2 \%$ had a diagnosis of TIA. This study adds data about yield and feasibility in real-world care, outside of a clinical trial. As in our study, patients in this single-centre study received their handheld ECG system soon after stroke, in this case a mean of 4.1 days from the stroke.

\section{Limitations}

Although the overall benefit of anticoagulation in patients with $\mathrm{AF}$ based on current risk assessment is unequivocal, the cause of stroke in an individual case is often hard to prove. We used a pragmatic approach based on current practice, even though a more detailed classification, based on imaging, of cryptogenic stroke has been suggested. ${ }^{27}$ The mechanisms of ischaemic stroke may be multifactorial. AF, especially recurrent and extended episodes, may cause remodelling of the atrial anatomy and structural changes. Even if AF is detected, consideration of other causes should not necessarily be ruled out. Therefore, an overall assessment of risk factors and treatment options is warranted in conjunction with a team-based approach, which is crucial to improve stroke management.

The incidence of AF in this trial is likely underestimated. The largest and most severe strokes often occur in the elderly, who are likely to be under-represented in clinical trials. However, the target population for this trial had to be able to handle the equipment and be followed by remote system in a outpatient setting after discharge. Patients with milder, less disabling stroke constitute an ideal group for prevention of recurrent stroke and death. In patients not eligible for thumb ECG, a device with adhesive electrodes would be an alternative. ${ }^{35}$ Although a thumb ECG is an attractive method of non-invasive AF detection, there remain some controversies with regard to anticoagulation for short-term AF. The duration of an AF episode captured by snapshot is unknown, but it is assumed to represent longer or multiple episodes. The potential benefits of anticoagulation therapy for shortterm AF would be challenging to study, because it would require long-term follow-up, demands a large sample size, a randomised controlled design and raises ethical concerns about withholding anticoagulation from stroke survivors.

\section{Future perspectives}

Guidelines already allow for prolonged monitoring of these patients: 'In patients who had a stroke, additional ECG monitoring by long-term non-invasive or implanted loop recorders should be considered to document silent AF' (class IIa recommendation, level of evidence B). ${ }^{7}$ Despite the landmark trial, ${ }^{11}$ current practice remains unchanged in that invasive monitoring is rarely used for AF detection in patients who had a stroke. The benefit of the thumb ECG in secondary stroke prevention has been demonstrated by our study, in addition to previous efforts in this setting. Yet, cost effectiveness in this setting from both healthcare and societal viewpoint remains to be elucidated.

Furthermore, a large-scale, multicentre, international, prospective observational trial is welcome in order to confirm diagnostic yield, and would allow for subgroup analyses and assessment of predictors for increased probability of AF. Artificial intelligence is tempting to consider in order to personalise evaluations, based on the presence of well-known markers of AF such as extrasystoles, clinical 
risk factors and possibly laboratory markers. Moreover, the feasibility of different devices and the length of evaluation time need to be further refined and adapted to possible subgroups of patients who had a stroke. In addition to advances in technology, the availability of devices as an integral part of stroke assessment needs to be increased.

\section{CONCLUSION}

In patients with cryptogenic stroke, $9 \%$ have $\mathrm{AF}$ detected using chest and thumb ECG two times per day over a period of 4 weeks. In many stroke survivors this is a feasible approach, which can potentially protect them from recurrent stroke by allowing for prompt initiation of NOAC treatment.

Acknowledgements The authors acknowledge editing by Jo Ann LeQuang of LeQ Medical who reviewed the manuscript for American English. Adrian Kling, Titti Lundgren, Ulf Tossman, Magnus Samuelsson and Philip Siberg from Coala Life are acknowledged for support regarding the Coala Life Monitor. The authors also wish to thank the staff at the stroke unit in Gävle and Hudiksvall for their participation in the study.

Contributors PM-idea, design, data collection, analyses and interpretation, administration, supervision, project management, and writing of the manuscript. $\mathrm{AL}$ - data collection and critical revision. GM—design, data collection, analyses and interpretation, administration, project co-management, and critical revision.

Funding Region Gävleborg funded this research project (grant number 20170220) and Coala Life provided free product Coala Heart Monitor during the study period.

Competing interests The project received free product from Coala Life. Outside this project: PM has received speaker fees or grants from Abbott, Alnylam, Bayer, AstraZeneca, Boehringer-Ingelheim, Internetmedicin, Lilly, MSD, Novo Nordisk, Octopus Medical, Orion Pharma, Pfizer, Vifor Pharma and Zoll. AL has received speaker fees from Pfizer. GM has received speaker fee from Alnylam, MSD and Internetmedicin.

Patient and public involvement Patients and/or the public were not involved in the design, or conduct, or reporting, or dissemination plans of this research.

Patient consent for publication Not required.

Ethics approval The study was approved by the Regional Ethical committee in Uppsala (Dnr 2017/321).

Provenance and peer review Not commissioned; externally peer reviewed.

Data availability statement № data are available. No additional data sharing available.

Open access This is an open access article distributed in accordance with the Creative Commons Attribution Non Commercial (CC BY-NC 4.0) license, which permits others to distribute, remix, adapt, build upon this work non-commercially, and license their derivative works on different terms, provided the original work is properly cited, appropriate credit is given, any changes made indicated, and the use is non-commercial. See: http://creativecommons.org/licenses/by-nc/4.0/.

\section{ORCID iD}

Peter Magnusson http://orcid.org/0000-0001-7906-7782

\section{REFERENCES}

1 Roth GA, Johnson C, Abajobir A, et al. Global, regional, and national burden of cardiovascular diseases for 10 causes, 1990 to 2015. J Am Coll Cardiol 2017;70:1-25.

2 Go AS, Mozaffarian D, Roger VL, et al. Executive summary: heart disease and stroke statistics--2014 update: a report from the American Heart Association. Circulation 2014;129:399-410.

3 Andersson T, Magnuson A, Bryngelsson I-L, et al. All-Cause mortality in 272,186 patients hospitalized with incident atrial fibrillation 19952008: a Swedish nationwide long-term case-control study. Eur Heart J 2013;34:1061-7.
4 Hart RG, Pearce LA, Aguilar MI. Meta-Analysis: antithrombotic therapy to prevent stroke in patients who have nonvalvular atrial fibrillation. Ann Intern Med 2007;146:857-67.

5 Ruff CT, Giugliano RP, Braunwald E, et al. Comparison of the efficacy and safety of new oral anticoagulants with warfarin in patients with atrial fibrillation: a meta-analysis of randomised trials. Lancet 2014;383:955-62.

6 Kernan WN, Ovbiagele B, Black HR, et al. Guidelines for the prevention of stroke in patients with stroke and transient ischemic attack: a guideline for healthcare professionals from the American heart Association/American stroke association. Stroke 2014:45:2160-236.

7 Kirchhof P, Benussi S, Kotecha D, et al. 2016 ESC guidelines for the management of atrial fibrillation developed in collaboration with EACTS. Eur Heart J 2016;37:2893-962.

8 Kishore A, Vail A, Majid A, et al. Detection of atrial fibrillation after ischemic stroke or transient ischemic attack: a systematic review and meta-analysis. Stroke 2014;45:520-6.

9 Henriksson KM, Farahmand B, Åsberg S, et al. Comparison of cardiovascular risk factors and survival in patients with ischemic or hemorrhagic stroke. Int J Stroke 2012;7:276-81.

10 Grond M, Jauss M, Hamann G, et al. Improved detection of silent atrial fibrillation using 72-hour Holter ECG in patients with ischemic stroke: a prospective multicenter cohort study. Stroke 2013;44:3357-64.

11 Sanna T, Diener H-C, Passman RS, et al. Cryptogenic stroke and underlying atrial fibrillation. N Engl J Med 2014;370:2478-86.

12 Diamantopoulos A, Sawyer LM, Lip GYH, et al. Cost-Effectiveness of an insertable cardiac monitor to detect atrial fibrillation in patients with cryptogenic stroke. Int J Stroke 2016;11:302-12.

13 Savelieva I, Camm AJ. Clinical relevance of silent atrial fibrillation: prevalence, prognosis, quality of life, and management. J Interv Card Electrophysiol 2000;4:369-82.

14 Friberg L, Hammar N, Rosenqvist M. Stroke in paroxysmal atrial fibrillation: report from the Stockholm cohort of atrial fibrillation. Eur Heart J 2010;31:967-75.

15 Vanassche T, Lauw MN, Eikelboom JW, et al. Risk of ischaemic stroke according to pattern of atrial fibrillation: analysis of 6563 aspirin-treated patients in ACTIVE-A and AVERROES. Eur Heart $J$ 2015;36:281-8

16 Healey JS, Connolly SJ, Gold MR, et al. Subclinical atrial fibrillation and the risk of stroke. N Engl J Med 2012;366:120-9.

17 Van Gelder IC, Healey JS, Crijns HJGM, et al. Duration of devicedetected subclinical atrial fibrillation and occurrence of stroke in ASSERT. Eur Heart J 2017;38:1339-44.

18 Boriani G, Glotzer TV, Santini M, et al. Device-detected atrial fibrillation and risk for stroke: an analysis of $>10,000$ patients from the SOS AF project (Stroke preventiOn Strategies based on Atrial Fibrillation information from implanted devices). Eur Heart $J$ 2014;35:508-16.

19 Glotzer TV, Hellkamp AS, Zimmerman J, et al. Atrial high rate episodes detected by pacemaker diagnostics predict death and stroke: report of the atrial diagnostics ancillary study of the mode selection trial (most). Circulation 2003;107:1614-9.

20 Glotzer TV, Daoud EG, Wyse DG, et al. The relationship between daily atrial tachyarrhythmia burden from implantable device diagnostics and stroke risk: the trends study. Circ Arrhythm Electrophysiol 2009;2:474-80.

21 Gorenek B, Bax J, Boriani G, et al. Device-detected subclinical atrial tachyarrhythmias: definition, implications and managementan European heart rhythm association (EHRA) consensus document, endorsed by heart rhythm Society (HRS), Asia Pacific heart rhythm Society (APHRS) and Sociedad Latinoamericana de Estimulación Cardíaca Y Electrofisiología (SOLEACE). Europace 2017:19:1556-78.

22 Stroke Risk in Atrial Fibrillation Working Group. Independent predictors of stroke in patients with atrial fibrillation: a systematic review. Neurology 2007;69:546-54.

23 Sposato LA, Cipriano LE, Saposnik G, et al. Diagnosis of atrial fibrillation after stroke and transient ischaemic attack: a systematic review and meta-analysis. Lancet Neurol 2015;14:377-87.

24 Rizos T, Güntner J, Jenetzky E, et al. Continuous stroke unit electrocardiographic monitoring versus 24-hour Holter electrocardiography for detection of paroxysmal atrial fibrillation after stroke. Stroke 2012;43:2689-94.

25 Insulander P, Carnlöf C, Schenck-Gustafsson K, et al. Device profile of the Coala heart monitor for remote monitoring of the heart rhythm: overview of its efficacy. Expert Rev Med Devices 2020;17:159-65.

26 Dolmans LS, Lebedeva ER, Veluponnar D, et al. Diagnostic accuracy of the explicit diagnostic criteria for transient ischemic attack: a validation study. Stroke 2019;50:2080-5. 
27 Hart RG, Catanese L, Perera KS, et al. Embolic stroke of undetermined source: a systematic review and clinical update. Stroke 2017;48:867-72.

28 Magnusson $\mathrm{P}$, Koyi H, Mattsson G. A protocol for a prospective observational study using chest and thumb ECG: transient ECG assessment in stroke evaluation (tease) in Sweden. BMJ Open 2018;8:e019933.

29 Friberg L, Rosenqvist M, Lip GYH. Evaluation of risk stratification schemes for ischaemic stroke and bleeding in 182678 patients with atrial fibrillation: the Swedish atrial fibrillation cohort study. Eur Heart $J$ 2012;33:1500-10.

30 Healey JS, Oldgren J, Ezekowitz M, et al. Occurrence of death and stroke in patients in 47 countries 1 year after presenting with atrial fibrillation: a cohort study. Lancet 2016;388:1161-9.

31 Gladstone DJ, Spring M, Dorian P, et al. Atrial fibrillation in patients with cryptogenic stroke. N Engl J Med 2014;370:2467-77.
32 Poulsen MB, Binici Z, Dominguez $\mathrm{H}$, et al. Performance of short ECG recordings twice daily to detect paroxysmal atrial fibrillation in stroke and transient ischemic attack patients. Int J Stroke 2017;12:192-6.

33 Doliwa Sobocinski P, Anggårdh Rooth E, Frykman Kull V, et al. Improved screening for silent atrial fibrillation after ischaemic stroke. Europace 2012;14:1112-6.

34 Orrsjö G, Cederin B, Bertholds E, et al. Screening of paroxysmal atrial fibrillation after ischemic stroke: 48-hour Holter monitoring versus prolonged intermittent ECG recording. ISRN Stroke 2014;2014:1-6.

35 Lumikari TJ, Putaala J, Kerola A, et al. Continuous 4-week ECG monitoring with adhesive electrodes reveals $\mathrm{AF}$ in patients with recent embolic stroke of undetermined source. Ann Noninvasive Electrocardiol 2019;24:e12649. 